\title{
CORRECTION
}

View Article Online

View Journal | View Issue

Check for updates

Cite this: Chem. Commun., 2021,

57, 2571

DOI: $10.1039 / \mathrm{d} 1 \mathrm{cc} 90077 \mathrm{c}$

rsc.li/chemcomm

\section{Correction: Highly stable zinc metal anode enabled by oxygen functional groups for advanced $\mathrm{Zn}$-ion supercapacitors}

\begin{abstract}
Kangyu Zou, ${ }^{a}$ Peng Cai, ${ }^{a}$ Xinglan Deng, ${ }^{a}$ Baowei Wang, ${ }^{a}$ Cheng Liu, ${ }^{a}$ Zheng Luo, ${ }^{a}$ Xiaoming Lou, ${ }^{b}$ Hongshuai Hou, ${ }^{a}$ Guoqiang Zou*a and Xiaobo $\mathrm{Ji}^{\text {ac }}$
\end{abstract}

Correction for 'Highly stable zinc metal anode enabled by oxygen functional groups for advanced Zn-ion supercapacitors' by Kangyu Zou et al., Chem. Commun., 2021, 57, 528-531, DOI: 10.1039/D0CC07526D.

The authors regret that the incorrect structure of konjac glucomannan (KGM) was used for Fig. 1, Fig. S12 and the Graphical Abstract in the original article. The redundant oxygen atom at the end of the repeating unit in the bracket has been deleted and

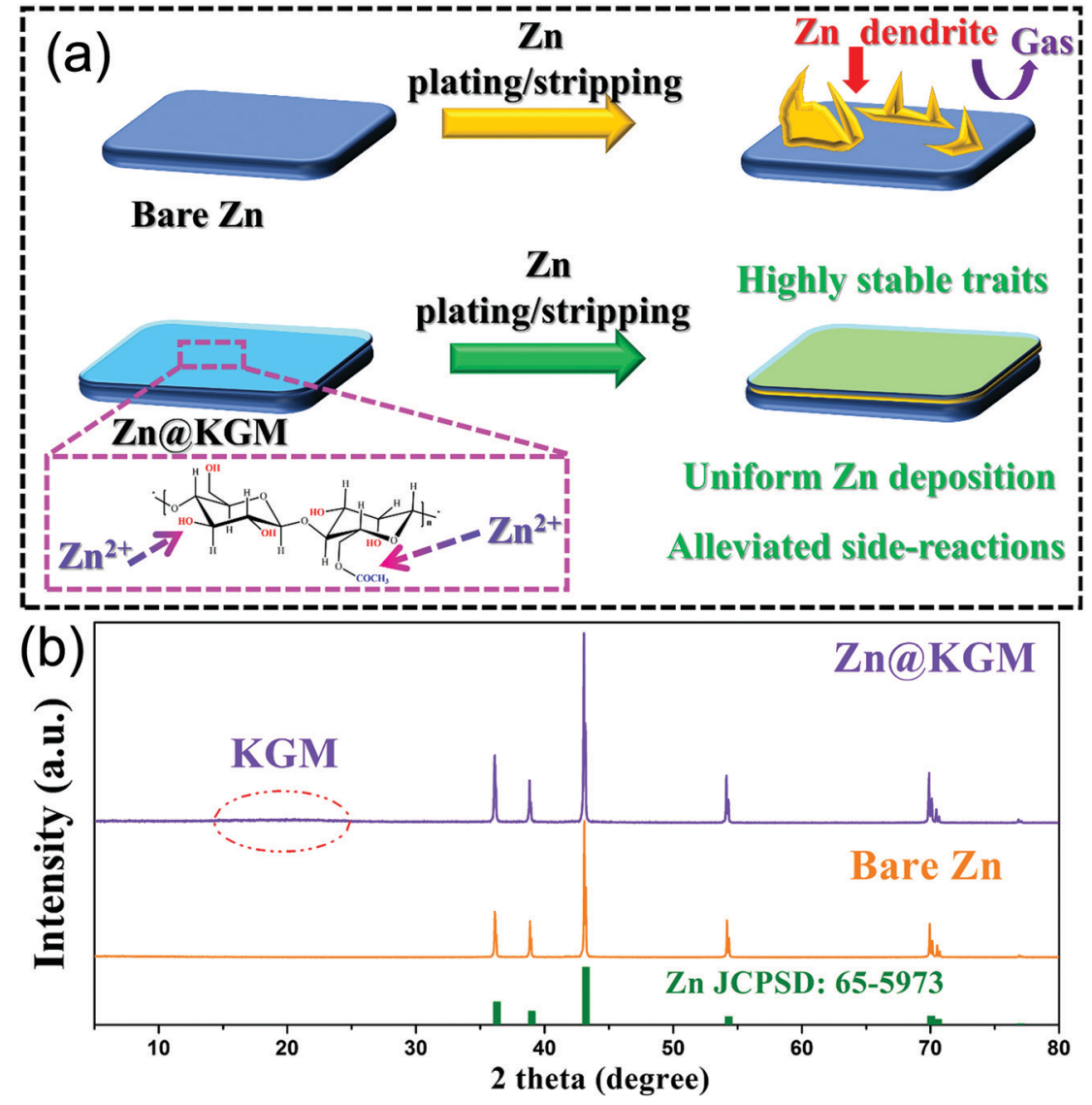

Fig. 1 (a) Schematic illustrations of the Zn stripping/plating processes of bare Zn and ZnaKGM anodes. (b) The XRD patterns of bare Zn and Zn@KGM anodes.

${ }^{a}$ College of Chemistry and Chemical Engineering, Central South University, Changsha, 410083, China. E-mail: gq-zou@csu.edu.cn

${ }^{b}$ School of Materials and Chemistry Engineering, Hunan Institute of Technology, 18 Henghua Rd, Hengyang, China

${ }^{c}$ School of Metallurgy and Chemical Engineering, Jiangxi University of Science and Technology, 86 Hongqi Road, Ganzhou 341000, China 
the position of the hydroxyl group in the glucose unit has been corrected to equatorial. Thus, the updated versions of Fig. 1 and Fig. S12 are displayed here, and the online Graphical Abstract has been updated with the corrected version. These errors do not affect any of the reported experimental results and discussions in the paper. The overall conclusions for the paper remain valid.

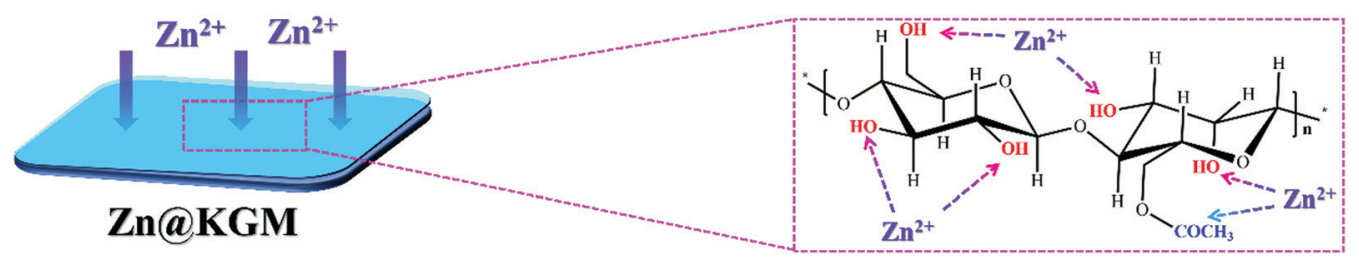

Fig. S12 Possible mechanism for stabilized $\mathrm{Zn}$ anode triggered by oxygen rich functional groups.

The Royal Society of Chemistry apologises for these errors and any consequent inconvenience to authors and readers. 